\title{
Hormigón liviano autocompactado de alta resistencia reforzado con fibras de acero. Análisis como material de refuerzo en vigas.
}

\author{
High strength steel fibers reinforced self-compacting \\ lightweight concrete. Analysis as reinforcement material in \\ beams.
}

\section{Doctorando:}

\author{
Ing. Alberto José Palacio \\ Centro de Investigación, Desarrollo y Transferencia de Materiales y Calidad (CINTEMAC), UTN F.R. Córdoba. \\ Grupo de Investigación de Ingeniería Civil Materiales y Ambiente (GIICMA), UTN F.R.Concordia. \\ apalacio@frcon.utn.edu.ar
}

\section{Director/es:}

\section{Director: Dra. Ing. Bárbara Belén Raggiotti, UTN - FRC. Codirector: Dra. Ing. Viviana Rougier, UTN- FRCU.}

\section{Resumen}

Frecuentemente resulta necesario reforzar elementos estructurales de un edificio existente. Es posible emplear como solución diferentes técnicas de refuerzo, entre ellas, el hormigón reforzado con fibras, debido a sus buenas propiedades de resistencia trabajando a flexión. Con el fin de aprovechar las ventajas que posee este material, adicionando una fácil ejecución con un mínimo agregado de peso propio y la ausencia de vibraciones en la estructura, se desarrolla el hormigón liviano de alta resistencia autocompactado y reforzado con fibras de acero (HLAARFA). Este proyecto tiene por objeto evaluar, la efectividad del HLAARFA como material de refuerzo de elementos sometidas a flexión, empleando materiales y sistemas de fabricación de nuestra región. Desde el punto de vista experimental, se estudiará el comportamiento a nivel material del HLAARFA, y luego se ensayarán modelos a escala de elementos estructurales de tipo vigas. Además, se espera simular el comportamiento de vigas reforzadas con este material mediante el diseño de un modelo numérico simplificado que permita calcular el momento flector resistente. La simulación se realizará mediante la implementación de un software de análisis por elementos finitos empleando la teoría para mezclas modificada. Se considerarán las diferentes fuentes de incertidumbres en los parámetros del modelo y se realizarán luego, estadísticas de los resultados obtenidos, los cuales serán contrastados con los datos experimentales.

Palabras claves: Hormigón alivianado, Fibras de acero, Refuerzo de vigas, Elementos finitos.

\footnotetext{
Abstract

Often it is necessary to reinforce structural elements of an existing building. It is possible to use different reinforcement techniques to solve this problem, including fiber reinforced concrete, due to its flexural strength properties. Due to the advantages that this material possesses, adding an easy execution with a minimum addition of own weight without the use of vibration in the structure, steel fiber reinforced high strength lightweight selfcompacting concrete is developed (SHLSCC). This project aims to assess the effectiveness of the HLAARFA as reinforcing material of elements subjected to flexion, using materials and manufacturing systems in our region. The material-level behavior of HLAARFA will be studied, and then scale models of structural elements of beam type will be tested. The behavior of reinforced beams will be simulated by designing a simplified numerical model that allows the calculation of the resistant bending moment. The simulation will be carried out through use of finite element analysis software using the modified mixture theory. The different sources of uncertainties in the parameters of the model will be considered. Finally, statistics of the results obtained will be made and will be contrasted with the experimental data.
} 


\section{Introducción}

La reparación o refuerzo de elementos estructurales de hormigón en estructuras existentes es muy importante para extender su vida útil, de otro modo la estructura tendría que ser demolida y reconstruida. En general, el refuerzo y/o la reparación de estructuras es más económico comparado con su demolición y reconstrucción (Nishino \& Aoki, 2006). Además existen construcciones que por su valor histórico-cultural merecen ser conservadas.

Entre los distintos materiales que se pueden usar para refuerzo a flexión, el hormigón re-forzado con fibras de acero presenta propiedades mecánicas y de durabilidad que lo hacen atractivo para estas actividades. La adición de fibras mejora la resistencia a tracción y flexión, la ductilidad y la tenacidad (Conforti et al., 2019).

Al reforzar los elementos estructurales de hormigón armado, el propósito es aumentar su capacidad resistente con un mínimo agregado de peso propio y evitando vibraciones en la estructura, lo cual puede ser no deseado para la estructura existente y las que la rodean. Por lo tanto, un hormigón liviano de alta resistencia autocompactado y reforzado con fibras de acero - HLAARFA (Steel fiber reinforced high strength lightweight self compacting concrete SHLSCC), podría ser utilizado para reforzar vigas de hormigón armado a los efectos de mejorar su resistencia a flexión (Holschemacher et al., 2015).

El hormigón autocompactado (self-compacting concrete - SCC) es altamente fluido, sin producir segregación. Tiene la propiedad de llenar los moldes y encapsular las armaduras de refuerzo sin consolidación mecánica. No requiere de vibración y compactación y puede fluir en amplios espacios sin excesiva exudación ni segregación, lo que resulta en un ahorro de mano de obra y una economía en el hormigonado.

Para el comité de la American Concrete Institute (ACI), el hormigón estructural liviano es el hormigón que tiene a los 28 días un resistencia a la compresión de $17 \mathrm{MPa}$ y densidad de $1120-1920 \mathrm{~kg} / \mathrm{m}^{3}$, constituido total o parcialmente por agregados livianos, mientras que los hormigones livianos de alta resistencia tienen a los 28 días una resistencia a la compresión de más de $40 \mathrm{MPa}$ (ACI 237R-07, 2007).

El uso de compuestos de polímeros reforzados con fibras (PRF) posee ventajas como refuerzo de elementos sometidos a flexión en cuanto a la relación resistencia/peso, resistencia a la corrosión, fácil y rápida colocación y mínimo cambio en la geometría de los miembros reforzados (Iqbal et al., 2016). Sin embargo, existen algunas desventajas importantes en el uso de PRF tales como el costo del "pegamento" epoxy, la no posibilidad de colocación en superficies húmedas y a bajas temperaturas, la falta de permeabilidad que puede ser dañina para la estructura de hormigón y la dificultad para realizar evaluación de posibles daños en los elementos de hormigón ocultos bajo una lámina de PFR. Para afrontar estos problemas, pueden ser utilizados morteros cementicios (Elsanadedy et al., 2013).

Shah y Ribakov (2011) demostraron que las propiedades mecánicas del hormigón de alta resistencia son mejoradas con el agregado de fibras en él. Las fibras son cortas para evitar problemas de trabajabilidad y una inadecuada distribución. El agregado de fibras de acero de alta resistencia al hormigón resulta en una mejor ductilidad y mayor capacidad de carga resistente, comparado con el hormigón con fibras de acero de resistencia normal en ausencia de armadura principal de refuerzo (Holschemacher et al., 2014). El uso de una relación óptima resistencia/peso produce elementos con alta performance a flexión con un comportamiento elasto-plástico similar a los hormigones armados convencionales (Iskhakov et al., 2014). De manera similar, el agregado de fibras a los hormigones livianos aumenta la capacidad de carga resistente y el control de fisuración (Mohammadi et al., 2008).

También las fibras aumentan la resistencia del hormigón a cargas dinámicas y de impacto, reducen el ancho de fisuración y aumentan la resistencia del hormigón en tracción. Sin embargo, el agregado de fibras influye negativamente sobre la trabajabilidad del hormigón (Ding et al., 2008). Akcay y Tasdemir (2012) usaron fibras de acero de resistencia normal y alta con $6 \mathrm{~mm}$ y $30 \mathrm{~mm}$ de longitud para estudiar el comportamiento de SCC reforzado con fibras. Concluyeron que el agregado de fibras influye en la trabajabilidad y que esta influencia depende más de la geometría de las mismas más que de su resistencia. Ha sido comprobado que el agregado de fibras de $20 \mathrm{~mm}$ de longitud al SCC reduce su trabajabilidad haciendo difícil el paso a través de la armadura tradicional (Khaloo et al., 2014). Liao et al. (2013) usaron fibras de acero de extremos en gancho para producir hormigón autocompactado reforzado con fibras de alta performance con $600 \mathrm{~mm}$ de extensión de flujo (slump flow), rango de resistencia a la compresión entre 35-65 MPa y rango de tensión de tracción de 3.5-6.5 MPa.

Ma et al. (2013) reportaron un 19-42\% de mejora en la resistencia a compresión de diferentes hormigones con el agregado de $0-2 \%$ de fibras cortas de $13 \mathrm{~mm}$ de longitud y relación de aspecto o esbeltez (l/d) de 65 . Resultados de estudios usando fibras de acero con longitudes en el rango de 22-44 $\mathrm{mm}$ indican que, la resistencia a la compresión no se ve afectada por el agregado de fibras, sin embargo el modo de fallo a compresión cambia de frágil a dúctil con los especímenes manteniendo su integridad hasta el final del ensayo (Olivito \& Zuccarello, 2010). Algunos estudios recientes también informan una disminución en la resistencia a compresión del hormigón con el agregado de fibras. Khaloo et al. (2014) obtuvieron un 18.6\% y 7.5\% de disminución en la resistencia a compresión de resistencias media y alta respectivamente con el agregado de $2 \%$ en fracción de volumen de fibras de acero. Una investigación llevada a cabo con el fin de estudiar las propiedades de hormigones de muy alta resistencia reforzados con fibras de polipropileno resistentes y micro fibras polivinílicas indicó una clara reducción de la resistencia a compresión y del módulo de elasticidad (Abu-Lebdeh et al., 2012). 
De acuerdo a una investigación realizada con SCC reforzado con fibras (Pajak \& Ponikiewski, 2013) usando fibras de acero rectas $12.5 \mathrm{~mm}$ y de $30 \mathrm{~mm}$ con extremos conformados en gancho, se observó que el SCC se comporta en una forma similar que el hormigón convencional vibrado y muestra un incremento en la resistencia a la flexión con el aumento en el contenido de fibras. Olivitto y Zuccarello (2010) concluyeron que el agregado de fibras cortas produce un comportamiento de ablandamiento (softening), donde las deformaciones se localizan en una única fisura, mientras que con fibras largas, el hormigón exhibe un comportamiento de endurecimiento (hardening), dando lugar a un incremento de carga con fisuración múltiple. Hay un aumento en la resistencia a flexión del hormigón con aumento del contenido de fibras de acero con el máximo valor correspondiente a un contenido de $3.5 \%$ en volumen. Más allá de ese valor, un aumento en el contenido de fibras disminuye la resistencia a flexión (Vinayak \& Mangulkar, 2013).

A los efectos de optimizar el uso de este nuevo material y su aplicación como material de refuerzo, resulta de fundamental importancia conocer y poder determinar las propiedades mecánicas que caracterizan al HLAARFA. Por otra parte, para aplicaciones prácticas, más estudios deberían llevarse a cabo para investigar el comportamiento mecánico de los elementos estructurales reforzados con este tipo de hormigones.

La tesis se enmarca en un proyecto de investigación que pretende investigar el uso estructural de este hormigón especial, con el empleo de materiales y sistemas de fabricación utilizados en la Región Pampeana como una opción técnica y económicamente posible. Comprendiendo estudios a nivel material, el estudio de dosificaciones de HLARFA y caracterización del mismo; ensayos experimentales de modelos a escala y simulación numérica mediante análisis por elementos finitos. El trabajo numérico-experimental y las conclusiones desarrolladas pasarán a enriquecer la literatura existente en el tema en el ámbito científico. Así mismo la presente investigación abrirá nuevas alternativas tecnológicas en lo que hace al diseño de una técnica eficiente de evaluación, refuerzo y/o reparación de elementos estructurales requerida por diferentes causas.

\section{Materiales y métodos}

Para el desarrollo de esta tesis será necesario un estudio teórico, experimental y numérico computacional.

En primer lugar se realizará una búsqueda bibliográfica para la recopilación de antecedentes sobre:

- Hormigones livianos de alta resistencia autocompactados reforzados con fibras de acero (HLAARFA).

- Comportamiento mecánico de HLAARFA como material de refuerzo en elementos estructurales.

- Modelos numéricos computacionales para evaluación de comportamiento de elementos estructurales sometidos s flexión.

Por otro lado, el desarrollo experimental comprende el estudio de materiales y la dosificación del hormigón, como también la dosificación optima de fibras de acero y sus efectos en la resistencia a compresión, tracción, flexión, y comportamiento post-fisuración. Se trabajará con materiales locales y la realización de probetas se llevará a cabo en el Laboratorio de Tecnología del Hormigón (FRCon) y en el Laboratorio del CINTEMAC (FRC).

Las propiedades del hormigón de refuerzo que se estudiarán en el estado fresco serán la trabajabilidad y el comportamiento reológico. Se determinará del peso de la unidad de volumen (IRAM 1562) y el contenido de aire (IRAM 1602). Se evaluará la capacidad de autocompactabilidad mediante la determinación de la capacidadad de llenado, la resistencia a la segregación o estabilidad y la capacidad de fluir entre pequeñas aberturas-obstáculos. Para ello los métodos propuestos son el ensayo de extendido, el ensayo J-Ring o anillo japonés y el ensayo L-Box para evaluar la capacidad de llenado y de sortear obstáculos; y el ensayo V-Funnel como indicador de la viscosidad plástica. Mientras que los parámetros resistentes y de deformación estudiadas en estado endurecido serán resistencia a la compresión a 7, 28 y 56 días (IRAM 1546), la resistencia a tracción por compresión diametral a 7 y 28 días (IRAM 1658), determinación del módulo de elasticidad estático (ASTM C 469) y resistencia flexional. Además se cumplirán exigencias del Reglamento CIRSOC 201-2005.

Para establecer las características estructurales debido al empleo de las fibras de acero se empleará el fib Model Code 2010 (MC2010), el cual propone un método de diseño que se basa en resistencias residuales post-fisura. En particular se consideran fR1 y fR3 parámetros de rendimiento, obtenidos en pruebas de flexión de tres puntos en prismas de dimensiones $150 \mathrm{~mm}$ x $150 \mathrm{~mm}$ x $600 \mathrm{~mm}$, con entalla central y cargados al centro (EN 14651, 2005). Las vigas serán instrumentadas midiendo la flecha con relación al eje de la viga y en la parte inferior midiendo la apertura de fisura.

Complementariamente se desarrollarán ensayos de caracterización de las fibras de acero, determinación de orientación y cantidad (UNE-EN 14721:2006+A1) y extracción de las mismas (ensayos de "Pull Out"), cuyos resultados serán utilizados en las aplicaciones numéricas.

A partir de los resultados experimentales se elaborará una base de datos con valores de resistencias a compresión y tracción y resistencias residuales a tracción de los hormigones reforzados con fibras utilizados en la región, que amplíen el campo de conocimiento del comportamiento de este material. 
En una etapa siguiente, para analizar la efectividad del refuerzo con HLAARFA se ensayarán series de 15 vigas de dimensiones de $150 \mathrm{~mm} \times 175 \mathrm{~mm} \times 1500 \mathrm{~mm}$ de hormigón convencional y armadura tradicional de barras longitudinales y estribos.

De cada serie de vigas, se tomarán tres de ellas como "vigas de referencia" y serán ensayadas a flexión en cuatro puntos simplemente apoyadas, sin refuerzos, con el fin de comprobar la forma de falla de las mismas. De las otras doce vigas, seis serán dañadas (prefisuradas) mediante el ensayo de flexión hasta alcanzar un ancho máximo de fisuración de $0.5 \mathrm{~mm}$ y luego descargadas. Luego serán aplicadas las capas de refuerzo de hormigón liviano autocompactado de alta resistencia reforzado con fibras de acero en capas inferiores de $30 \mathrm{~mm}, 40 \mathrm{~mm}$ y $50 \mathrm{~mm}$ en cada serie respectivamente. Las otras 6 vigas serán reforzadas sin ser prefisuradas.

Posteriormente las 12 vigas reforzadas serán ensayadas a flexión. Se repetirá el procedimiento para la cuarta serie aunque en este caso el refuerzo será aplicado en forma de $U$ en una capa de $30 \mathrm{~mm}$. Para una mejor adherencia entre el hormigón de las vigas y el de refuerzo se realizará un cincelado manual sobre la superficie de las vigas, antes de la aplicación de las capas de refuerzo.

Con el fin de registrar curvas de carga-flecha en cada ensayo, se colocarán instrumentos de medición en cada lado de la viga para registrar las deflexiones verticales una vez aplicada la carga.

La simulación numérica, se realizará mediante la implementación computacional en un software de análisis por elementos finitos. Para modelar el hormigón reforzado con fibras se usará la teoría de mezcla modificada. Se considerará al material formado por una matriz de hormigón con fibras en tres direcciones ortogonales. Para el hormigón se utilizará un modelo de daño plástico modificado y para las fibras un modelo elastoplástico ortótropo con funciones de fluencia y endurecimiento que representan adecuadamente el comportamiento de las fibras cuando son arrancadas de la matriz. A los fines de obtener una mejor representación del ensayo real se realizarán simulaciones Monte Carlo considerando diferentes fuentes de incertidumbres en los parámetros (propiedades mecánicas del HLAARFA) y hacer luego estadísticas de los resultados obtenidos.

\section{Resultados}

Los resultados y/o avances alcanzados en la investigación son escasos debido a que la admisión al doctorado se obtuvo en abril de 2019 (Resolución CS № 519/2019); por lo que se ha logrado avanzar en la revisión bibliográfica y la actualización del estado del arte respecto al empleo estructural de hormigones especiales. Así como el análisis de la normativa internacional y nacional vigente.

Actualmente se comenzó con la selección, estudio y ensayo de materiales locales, analizando disponibilidades y sus aptitudes para realizar las primeras pruebas de elaboración y caracterización del HLAARFA.

\section{Conclusiones}

En base a la temática del trabajo se espera lograr una contribución en los siguientes aspectos:

- Análisis de la efectividad del hormigón liviano autocompactado de alta resistencia reforzado con fibras de acero como refuerzo en vigas sometidas a flexión, de forma tal de lograr bases para una mejor aplicación de las ventajas que ofrece este material compuesto recientemente desarrollado. Evaluación de su aplicación con el empleo de materiales y sistemas de fabricación utilizados en nuestra región como una opción técnica y económicamente posible.

- Realizar ensayos que muestren la eficiencia del sistema de refuerzo en lo que respecta al aumento o recuperación de la capacidad portante en vigas de hormigón. Describir y calibrar los modelos constitutivos para una simulación numérica y su correspondiente comparación con estos resultados experimentales.

- Obtener conclusiones que enriquezcan la bibliografía existente relacionada a la eficiencia de este sistema como técnica de reparación y refuerzo de vigas de hormigón armado convencional sometidas a flexión y desarrollar recomendaciones útiles para su diseño en nuestra región.

- Proponer herramientas y criterios de diseño que contribuyan al desarrollo de una normativa sobre el tema, planteando alternativas tecnológicas en el diseño de una técnica eficiente de evaluación, refuerzo y/o reparación de elementos estructurales.

\section{Referencias}

Abu-Lebdeh, T.M., Fini, E., Lumpkin, M. (2012). Flexural and tensile characteristics of micro fiber-reinforced very high strength concrete thin panels. American Journal of Engineering and Applied Sciences, 5(2), 184-197.

ACI 237R-07. (2007). Self-Consolidating Concrete, American Concrete Institute 440R-07. Norms (p. 348). 
Akcay, B., \& Tasdemir, M. A. (2012). Mechanical behavior and fiber dispersion of hybrid steel fiber reinforced selfcompacting concrete. Construction and Building Materials, 28, 287-293.

Conforti, A., Zerbino, R., \& Plizzari, G. A. (2019). Influence of steel, glass and polymer fibers on the cracking behavior of reinforced concrete beams under flexure. Structural Concrete, 20(1), 133-143.

Ding, Y., Liu, S., Zhang, Y., \& Thomas, A. (2008). The investigation on the workability of fibre cocktail reinforced selfcompacting high performance concrete. Construction and Building Materials, 22(7), 1462-1470.

Elsanadedy, H. M., Almusallam, T. H., Alsayed, S. H., \& Al-Salloum, Y. A. (2013). Flexural strengthening of RC beams using textile reinforced mortar - Experimental and numerical study. Composite Structures, 97, 40-55.

Holschemacher, K., Müller, T., Kieslich, H. (2014) Flexural behavior of high-strength concrete with combined steel fiber and bar reinforcement. Proceedings of the International Seminar on Advanced Concrete Technology and its Applications, 45-51.

Iqbal, S., Ali, A., Holschemacher, K., \& Bier, T. A. (2015). Mechanical properties of steel fiber reinforced high strength lightweight self-compacting concrete (SHLSCC). Construction and Building Materials, 98, 325-333.

Iqbal, S., Ali, A., Holschemacher, K., Bier, T. A., \& Shah, A. A. (2016). Strengthening of RC beams using steel fiber reinforced high strength lightweight self-compacting concrete (SHLSCC) and their strength predictions. Materials and Design, 100, 37-46.

Iskhakov, I., Ribakov, Y., Holschemacher, K., Müller, T. (2014) Experimental investigation of full scale two-layer reinforced concrete beams. Mech. Adv. Mater. Struct. 21, 273-283.

Khaloo, A., Raisi, E. M., Hosseini, P., \& Tahsiri, H. (2014). Mechanical performance of self-compacting concrete reinforced with steel fibers. Construction and Building Materials, 51, 179-186.

Liao, W.C., Chao, S.H., Park, S.Y., Naaman, A.E. (2013) Self-consolidating high performance fiber reinforced concrete: SCHPFRC. Fifth International RILEM Workshop on High Performance Fiber Reinforced Cement Composites (HPFRCC5) 293-302.

Ma, H. L., Cui, C., Li, X., \& Hu, S. L. (2013). Study on mechanical properties of steel fiber reinforced autoclaved lightweight shell-aggregate concrete. Materials and Design, 52, 565-571.

Mohammadi, Y., Singh, S. P., \& Kaushik, S. K. (2008). Properties of steel fibrous concrete containing mixed fibres in fresh and hardened state. Construction and Building Materials, 22(5), 956-965.

Nishino, M., \& Aoki, T. (2006). Nonlinear analysis and damage monitoring of a one-sided patch repair with delamination. Composite Structures, 73(4), 423-431.

Olivito, R. S., \& Zuccarello, F. A. (2010). An experimental study on the tensile strength of steel fiber reinforced concrete. Composites Part B: Engineering, 41(3), 246-255.

Pająk, M., \& Ponikiewski, T. (2013). Flexural behavior of self-compacting concrete reinforced with different types of steel fibers. Construction and Building Materials 47, 397-408.

Shah, A.A., Ribakov, Y. (2011). Recent trends in steel fibered high-strength concrete. Materials and Design. 32, 41224151.

Vinayak, B.J., Mangulkar, M.N. (2013) Flexural behavior of self-compacting high strength fiber reinforced concrete (SCHSFRC). Int. J. Eng. Res. Appl. (IJERA) 3 (4), 2503-2505. 